\title{
Expanding the Role of the Patient-Centered Outcomes Research Institute: Reauthorization and Facilitating Value Assessments
}

\author{
William V. Padula ${ }^{1,2} \cdot$ R. Brett McQueen ${ }^{3}(\mathbb{D}$
}

Published online: 5 October 2019

(c) The Author(s) 2019

\section{Introduction}

The Patient-Centered Outcomes Research Institute (PCORI) was established by the Patient-Protection and Affordable Care Act in 2010 with the goal of helping patients and providers make better healthcare decisions. As an independent nonprofit organization drawing on government funding, it has pioneered research into the comparative clinical effectiveness of treatments and various healthcare approaches. All this useful information stands it in good stead as PCORI comes up for congressional reauthorization after September 2019.

At the same time, Congress should not miss this opportunity to push PCORI to focus on value in the health system. Despite its many achievements, PCORI is not directly associated with economically meaningful improvements in health system efficiency. If PCORI can successfully generate timely and useful evidence on patient-centered effectiveness, it should also be able to facilitate meaningful value assessments that navigate away from low-value services, thereby reducing costs and improving access to effective healthcare. Within its current mandate, we offer four simple recommendations for PCORI to achieve this goal and make meaningful impacts on improving value and efficiency in the US healthcare system.

R. Brett McQueen

robert.mcqueen@cuanschutz.edu

1 Department of Pharmaceutical and Health Economics, School of Pharmacy, University of Southern California, Los Angeles, CA, USA

2 Leonard D. Schaeffer Center for Health Policy and Economics, University of Southern California, Los Angeles, CA, USA

3 Department of Clinical Pharmacy, Center for Pharmaceutical Outcomes Research (CePOR), Skaggs School of Pharmacy and Pharmaceutical Sciences, University of Colorado Anschutz Medical Campus, Aurora, CO, USA

\section{How Did We Get Here?}

The original intent of PCORI was to expand healthcare research into areas that could help people make informed healthcare decisions that improve delivery and outcomes. It would go where traditional research did not, into questions that patients face as they seek care. To cite just a few examples, PCORI has funded a study that compared the outcomes of antibiotics vs. surgery to treat appendicitis [1]. It determined directly from stroke survivors the outcomes that mattered most to them by comparing their experiences with warfarin vs. oral anticoagulants [2]. It has backed over 100 studies on a wide range of conditions, including cancer, heart disease, and multiple chronic illnesses, where shared decision making was a key component [3].

However, noticeably absent from PCORI's objectives has been an explicit method to thoroughly support an understanding of value. The cost-effectiveness analysis has been a standard bearer of health economics research for over three decades; yet, PCORI statutes prohibit application of this methodology. In fact, PCORI statutes explicitly prohibit funding the use of any economic evaluations that use the primary statistic of a cost-effectiveness analysis, the cost per quality-adjusted life-year (QALY), otherwise known as the incremental cost-effectiveness ratio. This statute also extends to any research "that discounts the value of a life because of an individual's disability", which implicates the QALY [4].

Over the last decade, there has been a significant shift in emphasis on value over volume in healthcare. The simplest expression of value is represented by the classic ratio of "quality" per "cost" [5]. Nelson et al. expanded on this expression of value to incorporate more dimensions of healthcare delivery and technology that make quality a discrete measure [6]. That is, quality is a function of safety, effectiveness, equality, and time, leading to an equation as follows:

Value $=($ safety + effectiveness + equality $) /($ time + cost $)$. 
Optimizing value in a health system means that safety, effectiveness, and equality should be maximized, while time and cost are minimized. In fact, maximizing the patient experience with respect to these key parameters may produce a sense of value in healthcare that is greater than the sum of individual parameters [7]. PCORI's current focus on clinical trials for safety and comparative effectiveness has not improved the overall value of healthcare that patients consume. PCORI is hamstrung on maximizing such improvements because it lacks an expressed role in generating evidence that can support value assessments. PCORI's funded projects need to be analyzed with cost (and therefore time) as well as equality to achieve a recognizable impact on improving healthcare delivery.

\section{Emerging Use of Value Assessments in the USA}

Since the formation of PCORI, US value assessment frameworks have emerged that help providers, payers, and patients understand the value of new healthcare interventions and ideally make better choices about their use [8]. Value frameworks differ slightly in their mission, but all include some level of clinical comparison to the current standard of care for a therapy or technology. Common approaches to value assessment, such as conventional cost effectiveness, are often criticized for taking a 'one-size-fits-all' perspective on value, and some criticism is warranted. There are multiple components of value and the QALY by definition does not capture all potential benefits of healthcare interventions [9].

However, there is a reason the 'one-size-fits-all' approach persists. Academics and value frameworks depend on evidence gathered in efforts to win regulatory approval for drugs or other therapies, mainly in phase III clinical trials. Value assessors then link the evidence to QALYs, attach drug prices and health complication costs, and forecast the potential value of therapies over longer run time horizons. This is plainly unsatisfactory because regulatory approval evidence by definition is designed to meet safety and efficacy benchmarks, not value or other quality benchmarks. However, at present, there is no other readily available source of evidence on healthcare effectiveness. This is where PCORI could make a difference.

\section{How Can PCORI Facilitate Value Assessments Within its Mandate?}

PCORI funds studies that estimate outcomes important to patients, caregivers, and providers. This evidence is designed for real-world decision making. Evidence funded by PCORI such as patient-reported outcomes, real-world resource utilization, and estimates of effectiveness, not just clinical trial efficacy, can be used in the numerator and denominator of cost-effectiveness analyses.

PCORI has the opportunity to make four relatively simple and practical refinements to its focus to drive more evidence on value in healthcare. These recommendations include greater communication with stakeholders focused on value, expanding and facilitating the use of existing and future effectiveness data, ensuring timely accessibility of data for use in value studies, and developing methods that inform quantification of value. Adopting each of these recommendations can easily make PCORI-generated research relevant to value assessment efforts and advance the shift to value-based healthcare.

1. Communicate with value assessors by organizing a steering committee on value represented by Health Technology Assessment (HTA) organizations and researchers.

Refining PCORI's role to ensure generated evidence on effectiveness is useful in value estimation, and its agenda for priority research should align with that of value assessment developers. For example, PCORI could seek input from health economists and health policy researchers on key value questions in healthcare.

2. Expand and facilitate the use of effectiveness data available through PCORnet for incorporation into externally funded value studies.

One of PCORI's marquee efforts offers a catalog of robust evidence on healthcare effectiveness, the PCORnet [10]. PCORnet is a partnership of data sharing between academic medical centers and local community health clinics. PCORnet employs a common data model that links local data with the broader network for a streamlined research process. PCORnet states they are well equipped to conduct real-world evidence studies, pragmatic clinical trials, population health research, health systems research, and patient engagement studies. The information produced from PCORnet provides some of the richest information on effectiveness and safetytwo of the key inputs for value estimation.

3. Collaborate with PCORI-funded researchers to ensure external value assessors receive timely access to effectiveness data for inputs to value estimation.

As a complement to (2) above, to date, we know of no studies using PCORnet data as inputs to value estimation. While PCORI does not explicitly restrict access to PCORnet data for use in value estimation, the government mandate against formal cost-effectiveness studies is a signal to researchers that these data have restrictions on use. Simply communicating with researchers and value assessors that the effectiveness and safety data from PCORnet can be used as inputs into value assessment studies would expand the value assessment market. 
4. Identify and develop methods for quantifying non-traditional aspects of value.

A key challenge in value assessment is capturing the full range of treatment benefits that are meaningful from the patient and caregiver perspective. PCORI's mission can directly inform recent calls for research on measuring and incorporating non-traditional aspects of value (e.g., value of hope) into value assessments $[9,11]$. PCORI's past funding efforts have cultivated a robust literature base on patient-centered outcomes. Moving forward, PCORI can expand this effort to directly fund research on methods that support measurement and incorporation of non-traditional aspects of value.

Currently, no other US government entity funds studies that generate rich real-world effectiveness and utilization data. Encouraging the generation of evidence that can support value assessments and removing restrictions on externally funded or non-funded researchers using evidence generated by PCORI are important steps to embedding value assessments in American healthcare.

Author Contributions WVP and RBM contributed equally to this work according to the International Committee of Medical Journal Editors guidelines.

\section{Compliance with Ethical Standards}

Funding No sources of funding were received for the preparation of this editorial.

Conflict of interest William V. Padula and R. Brett McQueen received an unrestricted foundation grant from PhRMA to their respective universities. In addition, William V. Padua is a principal consultant for Monument Analytics, and R. Brett McQueen consults with Systematic Healthcare Directions; these consulting relationships have no perceived conflicts of interest to the related work.

Open Access This article is distributed under the terms of the Creative Commons Attribution-NonCommercial 4.0 International License (http://creativecommons.org/licenses/by-nc/4.0/), which permits any noncommercial use, distribution, and reproduction in any medium, provided you give appropriate credit to the original author(s) and the source, provide a link to the Creative Commons license, and indicate if changes were made.

\section{References}

1. Minneci PC, Hade EM, Lawrence AE, et al. Multi-institutional trial of non-operative management and surgery for uncomplicated appendicitis in children: design and rationale. Contemp Clin Trials. 2019;83:10-7.

2. Xian Y, Xu H, O'Brien EC, et al. Clinical effectiveness of direct oral anticoagulants vs warfarin in older patients with atrial fibrillation and ischemic stroke: findings from the patient-centered research into outcomes stroke patients prefer and effectiveness research (PROSPER) study. JAMA Neurol. 2019. https://doi. org/10.1001/jamaneurol.2019.2099 (epub ahead of print).

3. Partnership to Improve Patient Care. Primer: PCORI background, funding streams, and reauthorization. Washington, DC: PIPC; 2018. http://www.pipcpatients.org/blog/primer-pcori-background -funding-streams-and-reauthorization. Accessed 16 Sep 2019.

4. The Patient Protection and Affordable Care Act. 2010. PL 111-148.

5. Padula WV, Lee KKH, Pronovost PJ. Using economic evaluation to illustrate value of care for improving patient safety and quality: choosing the right method. J Patient Saf. 2017. https://doi. org/10.1097/pts.0000000000000410 (epub ahead of print).

6. Nelson EC, Batalden PB, Godfrey MM, Lazar JS. Value by design. San Francisco (CA): Jossey-Bass; 2011.

7. Torrance GW, Thomas WH, Sackett DL. A utility maximization model for evaluation of health care programs. Health Serv Res. 1972;7(2):118-33.

8. Neumann PJ, Cohen JT. Measuring the value of prescription drugs. N Engl J Med. 2015;373(27):2595-7.

9. Lakdawalla DN, Doshi JA, Garrison LP Jr, Phelps CE, Basu A, Danzon PM. Defining elements of value in health care; a health economics approach: an ISPOR Special Task Force Report [3]. Value Health. 2018;21(2):131-9.

10. The National Patient-Centered Clinical Research Network. About PCORnet. Washington, DC: PCORnet; 2019. https://pcornet.org/. Accessed 16 Sep 2019.

11. Diaby V, Ali AA, Montero AJJP-O. Value assessment frameworks in the United States: a call for patient engagement. Pharmacoecon Open. 2019;3(1):1-3. 